\title{
AVALIAÇÃO DA LIBERAÇÃO DE FLÚOR DE ALGINATOS ODONTOLÓGICOS
}

\author{
EVALUATION OF FLUORIDE RELEASE OF DENTAL ALGINATES
}

\author{
Matheus Melo Pithon" \\ Rogério Lacerda dos Santos * \\ Alline Birra Nolasco Fernandes "* \\ Edna Lúcia Couto Oberosler \\ Delmo Santiago Vaitsman
}

\section{RESUMO}

Introdução: O propósito do presente trabalho é avaliar a liberação de flúor de alginatos de uso odontológico. Materiais e Métodos: Foram avaliadas cinco marcas de alginatos divididos em cinco grupos: Jeltrate, Alga Gel, Printer Gel,Ava Gel e New Print. Os alginatos foram manipulados seguindo as orientaçóes dos fabricantes. Após isso, seguiu-se à confecção dos corpos de prova, utilizando-se moldes de silicone nas dimensôes de $4 \mathrm{~mm}$ de diâmetro e $4 \mathrm{~mm}$ de altura. Após geleificação, os corpos de prova foram removidos dos moldes e inseridos em recipiente com $10 \mathrm{ml}$ de água milliQ, por $2 \mathrm{~min}$. A liberação de flúor foi medida, através de eletrodo íon seletivo conectado a um analisador de íons. Resultados: $\mathrm{O}$ Ava Gel liberou menor quantidade de flúor e foi diferente estatisticamente dos grupos Alga Gel e New Print $(\mathrm{P}<0,05)$. O New Print que liberou mais apresentou similaridade estatística apenas com o grupo Alga Gel que por sua vez foi similar estatisticamente ao Jeltrate e Printer Gel $(P>0.05)$. Conclusōes: Pode-se concluir com a realização deste trabalho que todos os alginatos testados liberaram flúor, um em mais e outros em menor quantidade.

DESCRITORES: Intoxicação por Flúor • Materiais para Moldagem Odontológica • Materiais Dentários.

\section{ABSTRACT}

Introduction: The purpose of this study is to evaluate the release of fluoride from alginate for dental use. Materials and Methods: Seventy-five brands of alginate divided into five groups: Jeltrate, Alga Gel, Printer Gel, Ava Gel and New Print. The alginate were handled following the guidelines of the manufacturers. After that begin the making of the specimes using silicone molds of the dimensions of $4 \mathrm{~mm}$ in diameter and $4 \mathrm{~mm}$ in height. After cured the samples were removed from the molds and placed in container with 10 $\mathrm{ml}$ of water milliQ, for $2 \mathrm{~min}$. The release of fluoride was measured by ion selective electrode connected to an analyzer of ions. Results: The Ava Gel smaller amount of fluoride released and was statistically different groups of Alga and New Gel Print $(\mathrm{P}<0.05)$. The New Print that released more statistics showed similarity only to the group Alga Gel which in turn was statistically similar to Jeltrate and Gel Printer $(P>0.05)$. Conclusions: All alginate tested released fluoride, and one more in a lesser amount.

DESCRIPTORS: Fluoride Poisoning • Dental Impression Materials • Dental Materials.

* Especialista em Ortodontia pela Universidade Federal de Alfenas - Unifal; Mestre em Ortodontia pela Universidade Federal do Rio de Janeiro - UFRJ;

Doutorando em Ortodontia pela Universidade Federal do Rio de Janeiro - UFRJ.

** Cirurgiã dentista graduada pela Universidade Federal do Rio de Janeiro - UFRJ.

*** Bacharelado e Licenciatura em Ciências Biológicas pela Fundação Técnico Educacional Souza Marques; Técnica Química - LaDA - Intítuto de Química/ UFRJ.

**** Doutor em Química pela Pontíficia Universidade Católica-Puc-RJ; professor adjunto da Universidade Federal do Rio de Janeiro -UFRJ; coodenador e do Laboratório de Desenvolvimento Analítico (LaDa-IQ/UFRJ). 


\section{INTRODUÇÃO}

O alginato ou hidrocoloide irreversível é um dos materiais de moldagem mais aceitos e utilizados na Odontologia (Rodrigues et al. ${ }^{11}$ 2009, Schroeder ${ }^{12}$ 2008). Os fabricantes produzem o pó de alginato contendo vários componentes, com diferentes finalidades. A terra diatomácea e o óxido de zinco atuam como carga, influenciando as propriedades físicas e o tempo de presa do gel e o sulfato de cálcio é empregado como ativador da reação. Os fluoretos, como o fluoreto de titânio, são acrescentados à fórmula como aceleradores de presa e ainda asseguram que a superfície do gesso quando vazado sobre o molde tenha dureza e densidade adequadas (Lee et al. ${ }^{9}$ 2004, Anusovice ${ }^{1}$ 2005).

O flúor é um dos oligoelementos mais conhecidos pelo importante papel que desempenha na prevenção e controle da cárie. No entanto, a ingestão acima da dose recomendada ao dia $(0,05$ a $0,07 \mathrm{ppm}$ de $\mathrm{F}-)$ pode causar intoxicação (Hattab e Frostell ${ }^{7} 1980$ ). Os principais problemas de intoxicação crônica pelos fluoretos são a fluorose dentária e a osteosclerose (Pak et al. ${ }^{10}$ 1995, Whitferd ${ }^{15}$ 1997).

Os pacientes podem ser expostos aos fluoretos dos alginatos por meio da moldagem e pela ingestáo acidental de pedaços do material (Hattab e Frostell7 1980). Nesse procedimento o material pode permanecer até cinco minutos na cavidade bucal para que ocorra sua completa geleificação. Dessa forma, a quantificação do flúor presente nos alginatos comercialmente disponíveis no mercado é de suma importância para que o clínico possa trabalhar com maior segurança.

Baseado nessa premissa, o presente artigo tem como objetivo avaliar a liberação de flúor de 5 diferentes marcas de alginatos odontológicos.

\section{MÉTODOS}

\section{Alginatos avaliados}

A amostra foi composta de cinco diferentes alginatos divididos em cinco grupos: Jeltrate (Dentsply, Petrópolis, Brasil, Lote 971244), Alga Gel (Technew, Rio de Janeiro, Brasil, Lote 08276), Printer Gel (Euronda, Magé, Brasil, Lote 012/06), Ava Gel (Dentsply, Petrópolis, Brasil, Lote024471A ) e New Print (Technew, Rio de Janeiro, Brasil, Lote 08063).

\section{Confecção dos corpos de prova}

Para confecção dos corpos de prova, foram utilizados moldes de silicone nas dimensóes de $4 \mathrm{~mm}$ de diâmetro e $4 \mathrm{~mm}$ de altura. Inicialmente foram colocados 2,25g de alginato (pó) em um grau de borracha e acrescentados 4,5 $\mathrm{ml}$ de água destilada, manipulando-se por um minuto, de acordo com a especificação do fabricante. Uma vez manipulado, o alginato foi inserido dentro dos moldes com auxílio de espátula de plástico, evitando-se, assim, a formação de bolhas. A superfície dos corpos de prova foi coberta com lâminas de vidro sob pressão digital, proporcionando planificação da superfície do material até completa geleificação.

Após geleificação, o alginato foi removido dos moldes e inserido em recipientes tipo becker contendo $10 \mathrm{ml}$ de água milliQ, por 2 minutos. Passado esse período os corpos de prova foram removidos, ficando a água milliQ para ser analisada quanto à concentração de flúor presente nesta, que foi liberada pelos alginatos.

\section{Concentraçáo de fluoreto}

A determinação da concentração de fluoreto nos alginatos foi realizada no Laboratório de Química analítica da Universidade Federal do Rio de Janeiro-UFRJ, pelo método do eletrodo seletivo de fluoreto. $\mathrm{O}$ método baseia-se na medida de diferença de potencial do eletrodo em contato com a amostra. As determinaçóes de fluoreto nas diferentes amostras foram realizadas por potenciometria direta, utilizando-se o eletrodo seletivo de fluoreto (Orion, modelo $96-09$ ), usando-se como ajuste de forçaiônica e de $\mathrm{pH}$, o tampão citrato $0,5 \mathrm{~mol} / \mathrm{l}, \mathrm{pH} 5,5$, na proporção de 1:1 amostra/tampão. A concentração de fluoreto nas amostras (x) foi determinada pelo valor do respectivo potencial obtido (y) e empregando a equação $\mathrm{da}$ reta $\mathrm{y}=\mathrm{b}+\mathrm{a} \log \mathrm{x}$, obtida por regressão linear de leituras em triplicatas de soluçóes diluídas convenientemente, a partir de uma soluçáo-padrão de fluoreto, sendo a o coeficiente angular da reta e $\mathbf{b}$ o coeficiente linear.

A curva de calibração foi obtida a partir de leituras

Tabela 1. Valores médios e desvio padrão da liberação de flúor dos materiais avaliados em $\mu \mathrm{g} / \mathrm{cm} 2$

\begin{tabular}{ccc}
\hline \hline Grupos & Média (DP) & Estatística* $^{*}$ \\
\hline Jeltrate & $79,61(31,24)$ & $\mathrm{AC}$ \\
Alga Gel & $371,97(155,2)$ & $\mathrm{AB}$ \\
Printer Gel & $133,35(43,86)$ & $\mathrm{AC}$ \\
Ava Gel & $21,57(4,55)$ & $\mathrm{C}$ \\
New Print & $640,44(304,12)$ & $\mathrm{B}$ \\
\hline \hline DP $=$ Desvio padrão; & \\
${ }^{*}=$ Letras iguais indica ausência de diferenças estatísticas $(\mathrm{P}>0.05)$.
\end{tabular}


de diferença de potencial $(\mathrm{mV})$ de soluçóes-padróes de fluoreto de sódio com concentraçóes que variavam de 1 a $100 \mu \mathrm{g} / \mathrm{g}$.

\section{Análise estatística}

As analises estatísticas foram realizadas com auxílio do programa SPSS 13.0 (SPSS Inc.,Chicago, Illinois). Pela análise estatística descritiva, a média e desvio-padrão foram calculados para os grupos avaliados. Os valores da quantidade de flúor liberado foram submetidos à análise de variância (ANOVA) para determinar se havia diferenças estatísticas entre os grupos e, posteriormente, ao teste de Tukey.

\section{RESULTADOS}

Os resultados obtidos quanto à liberação de flúor dos alginatos avaliados estáo descritos na Tabela 1.

O grupo New Print apresentou a maior liberação de flúor seguido do grupo Alga Gel, Printer Gel, Jeltrate e Ava Gel. O Ava Gel foi diferente estatisticamente dos grupos Alga Gel e New Print $(\mathrm{P}<0,05)$. O New Print apresentou similaridade estatística apenas com o grupo Alga Gel que, por sua, vez foi similar estatisticamente ao Jeltrate e Printer Gel $(\mathrm{P}>$ 0.05) (Figura 1).

\section{DISCUSSÃO}

O potencial tóxico do fluoreto tem sido muito discutido na literatura. $\mathrm{Na}$ toxicidade crônica o fluoreto altera o mecanismo homeostático, afetando o metabolismo do cálcio (Das et al. ${ }^{5}$ 1994). Foi verificada insuficiência renal crônica em pessoas que ingeriam água mineral (dois a quatro litros/ dia) contendo 8,5 ppm de F- durante 20 anos. No entanto, outros estudos epidemiológicos não encontraram doenças renais pela exposição por meio da água de consumo com até oito ppm (Lanz et al. ${ }^{8}$ 1987).
Uma alteração importante causada pela ingestão de elevada e longo prazo de fluoreto é a fluorose dental, pois o fluoreto tem efeito sobre a nucleação dos ameloblastos e sobre o crescimento cristalino em todas as fases de formação do esmalte, além de efeitos sobre a homeostase do cálcio (Warren et al. ${ }^{14}$ 2003). A ingestáo de flúor em doses elevadas pode levar também a fluorose esquelética, pois causa alteração no acréscimo ou absorção do tecido ósseo, afetando o metabolismo mineral ósseo (Chavassiex3 1990, Gupta et al. ${ }^{6}$ 2001).

Conforme Cury (Cury et al. ${ }^{9}$ 2004), mesmo dentro do padrão considerado ótimo (0,7 ppm no Brasil) poderá haver fluorose clinicamente aceitável. Para concentraçôes maiores, agrava-se o problema compromentendo-se cada vez mais a estética e até mesmo a função dos dentes. Em concentrações oito vezes acima do padrão "ótimo", tem-se, do ponto de vista de saúde geral, comprometimento ósseo, caracterizando-se radiograficamente por radiopacidade óssea sem qualquer sintoma clínico.

Além do flúor estão presentes na água creme dental, fios dentais, soluções de bochecho. O flúor também é parte constituinte dos alginatos de uso odontológico

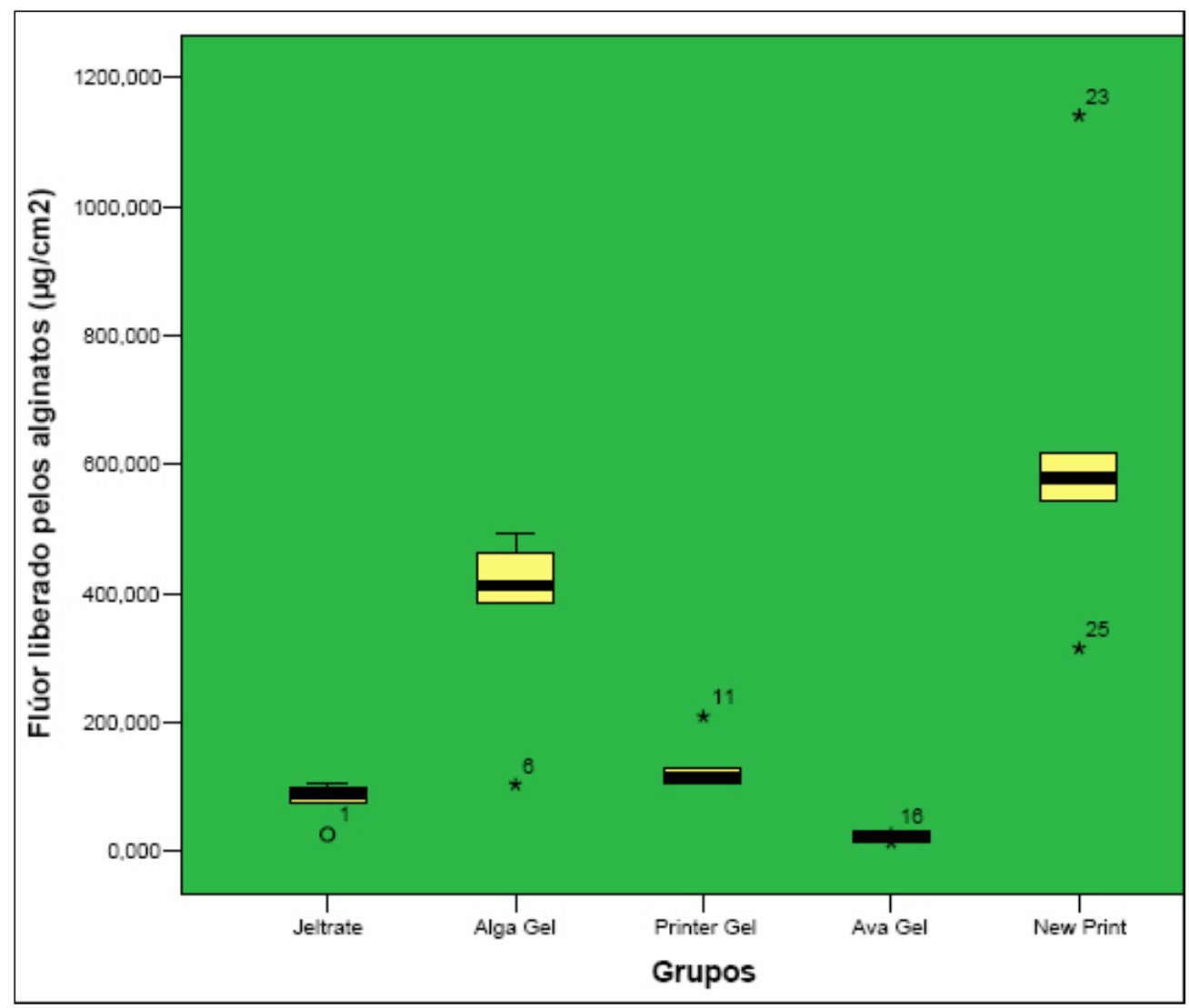


(Lee et al. ${ }^{9}$ 2004).

Os fluoretos como o fluorsilicato de sódio (Buchan et al. ${ }^{2}$ 1996), o fluoreto de potássio e o fluoreto de titânio são acrescentados à fórmula como aceleradores de presa do gesso, assegurando que a sua superfície tenha dureza e densidade adequadas (Anusavici ${ }^{1} 2005$ ).

Dessa forma o presente artigo se propóe avaliar a liberação de flúor de moldes de diferentes marcas de alginatos de uso odontológico.

A liberação de fluoreto foi analisada in vitro, em água milliQ. Os passos de confecção dos corpos de prova foram similares ao que ocorre em um ato normal de moldagem. Após geleificação do material, os corpos de prova foram inseridos em recipiente contendo $10 \mathrm{ml}$ de água milliQ que serviu de veículo para liberação de flúor neles presente. Após esse passo, a água milliQ foi analisada e verificou-se a quantidade de flúor liberada.

Os resultados demonstraram alta concentração de flúor nos alginatos New Print, seguidos do Alga Gel em menor quantidade, entretanto bem acima dos níveis liberados pelo Ava Gel e Jeltrate que foram os que menos liberaram flúor.

A importância de se saber esses níveis é que, com isso, o profissional pode conhecer melhor os materiais que está utilizando, propiciando um atendimento com maior segurança, evitando-se intoxicaçóes agudas e o agravamento de intoxicações crônicas.

Segundo a literatura, cuidados especiais devem ser tomados ao realizar moldagem com alginato em crianças. Esses cuidados seriam: após a moldagem, jogar água na boca do paciente e pedir para que ele cuspa, além de um exame cuidadoso da boca para que pedaços de material sejam removidos (Shulman e Wells ${ }^{13}$ 1997).

Vale ressaltar que o sucesso na clínica odontológica não envolve somente o domínio da técnica, mas também requer a aplicação das normas de biossegurança e a preocupação com as consequências locais e sistêmicas dos materiais dentários utilizados.

\section{CONCLUSÕES}

Pode-se concluir com a realização deste trabalho que:

- todos os moldes de alginatos liberaram flúor durante o ato de moldagem;

- alginato da marca New Print liberaram 30 x mais flúor que o Ava Gel.

\section{REFERÊNCIAS}

1. Anusavice KJ. Phillips, materiais dentários, $11^{\mathrm{a}}$ ed.; Rio de Janeiro: Guanabara, 2005.

2. Buchan S, Peggie RW. Role of ingredients in alginate impression compounds. J Dent Res. 1966 Jul- Aug; 45(4):1120-9.

3. Chavassieux P. Bone effects of fluoride in animal models in vivo. A review and a recent study. J Bone Miner Res. 1990 Mar; 5 Suppl 1:S95-9.

4. Cury JA, Tenuta LM, Ribeiro CC, Paes Leme AF. The importance of fluoride dentifrices to the current dental caries prevalence in Brazil. Braz Dent J. 2004;15(3):167-74 Epub 2005 Mar 18. Review.

5. Das TK, Susheela AK, Gupta IP, Dasarathy S, Tandon RK. Toxic effects of chronic fluoride ingestion on the upper gastrointestinal tract. J Clin Gastroenterol. 1994 Apr; 18(3):194-9.
6. Gupta SK, Khan TI, Gupta RC, Gupta AB, Gupta $\mathrm{KC}$, Jain $\mathrm{P}$, et al. Compensatory hyperparathyroidism following high fluoride ingestion - a clinico biochemical correlation. Indian Pediatr. 2001 Feb; 38(2):139-46.

7. Hattab F, Frostell G. The release of fluoride from two products of alginate impression materials. Acta Odontol Scand. 1980;38(6):385-95.

8. Lantz O, Jouvin MH, De Vernejoul MC, Druet P. Fluoride-induced chronic renal failure. Am J Kidney Dis. 1987 Aug; 10(2):136-9.

9. Lee YK, Lim BS, Kim CW. Effect of fluoride addition on the properties of dental alginate impression materials. J Mater Sci Mater Med. 2004 Mar; 15(3): 219-24.

10. Pak CY, Zerwekh JE, Antich P. Anabolic effects of fluoride on bone. Trends Endocrinol Metab. 1995 Sep; 6(7):229-34. 
11. Rodriguez JM, Curtis RV, Bartlett DW. Surface roughness of impression materials and dental stones scanned by non-contacting laser profilometry. Dent Mater. 2009 Apr; 25(4): 500-5. Epub 2008 Nov 25.

12. Schroeder E. Dental impression wafers. J Am Dent Assoc. 2008 Mar; 139(3): 236; author reply 238, 242, 245.

13. Shulman JD, Wells LM. Acute fluoride toxicity from ingesting home-use dental products in children, birth to 6 years of age. J Public Health Dent. 1997 Summer; 57(3):150-8.
14. Warren JJ, Slayton RL, Yonezu T, Kanellis MJ, Levy SM. Interdental spacing and caries in the primary dentition. Pediatr Dent. 2003 Mar-Apr; 25(2): 10913.

15. Whitford GM. Determinants and mechanism of enamel fluorosis. Ciba Found Symp. 1997; 205: 22645 .

Recebido em: 12/2/2009

Aceito em: 1/10/2009 